\title{
Modelling of macrophage interactions in breast cancer by partial differential equations
}

\author{
Mohd Rashid Admon and Normah Maan* \\ Department of Mathematical Science, Faculty of Science, Universiti Teknologi Malaysia, 81310 UTM Johor Bahru, Johor, Malaysia \\ *Corresponding author: normahmaan@utm.my
}

\section{Article history}

Received 17 February 2017

Accepted 11 May 2017

\begin{abstract}
The recruitment of macrophages at the tumor sites is the earliest immune response takes place during tumor progression. In breast cancer, experimental studies reveals that the tumor cells are capable of taking advantage on the plasticity of macrophages. Tumor cells respond to epidermal growth factor, EGF that released by macrophages while macrophages respond to colony stimulating factor 1 , CSF1 that released by tumor cells. This chains continues and results a paracrine signalling loop. Consequently, tumor cells and macrophages will aggregate and invade to other tissues or organ. Tumor cells also receive their own signals, adding a new feature of interaction called autocrine signalling loop. By considering in vitro interactions, a system of partial differential equations that incorporate the saturating functions for secretion terms was developed. This functions describes the production of chemical signals saturates with increasing cell density. Stability analysis are then performed to investigate the conditions for aggregation. For a given average of cells density, the homogeneous steady state is non-trivial and the concentration of CSF-1 and EGF are produced in the saturated production. Stability results show that regions for instability are reduced, compared to previous model which assumes the production rates are linear with increasing cell density. Besides, the inclusion of autocrine signalling loop increase the occurrence of aggregation. Decreasing the production rates and chemotaxis sensitivity, together with increasing the decay rates are required to impede the aggregation from initiated. This results should provide valuable clinical suggestions in guiding medical experts during drug designs.
\end{abstract}

Keywords: Partial differential equations, macrophages, breast cancer, saturating functions, Stability region

(C) 2017 Penerbit UTM Press. All rights reserved

\section{INTRODUCTION}

Breast cancer is the most commonly diagnosed cancer type and a leading killer among women across the globe $[1,2,3]$. Great efforts have been done to improve the current understanding of the disease at many stages of its development. These include prevention, diagnosis, tumor progression and recurrence, treatment and therapeutic resistance [4]. The fact that it is a heterogeneous disease among different patient's cause's diagnosis and therapy problems to remain challenging [5].

In general, breast cancer arises from abnormal mammary stem cells that form tumor which lose their cell characteristics and behaviour [6]. As soon as the abnormal cell emerges, immune system is a crucial component in human body to perform their task. Experts have been widely use this biological fact since it is obviously realistic in human life. Mathematical approaches in analysing the interaction between immune and breast tumor cells can be found in previous literature [7$11]$.

However, recent knowledge of immune and breast tumor cells interaction (also implies in some type of cancer) have reveals the paradox of tumor immunology [12]. This issue have been controversy due to contradict nature of immune system. Macrophages are one of the innate immune system identified as the causes for poor prognosis in several cancer including breast cancer $[13,14,15]$.

Macrophages are parts of immune system that have diverse of functions, including in ecosystem of cancer $[14,15,16]$. In normal situation, they are required for homeostasis, tissue remodelling, angiogenesis and disposal of dead cells [14, 17-23]. Due to the unfavourable condition in cancer surrounding, their function may reverse to promote the growth of tumor. Several theories have been developed to differentiate the positive and negative functions of macrophages $[22,24]$. The presented work focuses on the negative role of macrophages.

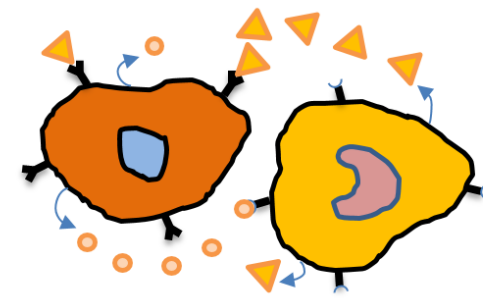

EGF Y

O CSF-1 ICSF-1R

Fig. 1 Interaction between tumor cells and macrophages.

The way of macrophages to carry out their functions is depending on the environmental cues $[13,14,17]$. When cancer develops, macrophages are manipulated by tumor cells which reverse the defence mechanism to negative action. This action is carried out in the form of communication via signalling molecule that trigger the tumor cells migrate from their primary sites [25-28]. 
Tumor cells was first release the colony stimulating factor, CSF-1 and receive by the receptor on macrophage, CSF-1R. This will activated the macrophages which triggered them to move to the higher concentration (chemotaxis) of CSF-1 and release epidermal growth factor, EGF. Tumor cell accept the EGF since they have epidermal growth factor receptor, EGFR) and chemotact towards the higher concentration of EGF. This chain continues and create a paracrine signalling loop which then both will aggregate [25]. The cooperative migration lead tumor to a distant sites by either intravasation or extravasation to form a secondary tumor [27].

The current study is motivated by Knutsdottir et al. [29]. They constructed the first model on the interaction between macrophages and tumor cells. They use a system of partial differential equation that consists of chemotaxis and reaction-diffusion equations. In other works, Elitas and Zeinali [30] investigated the glioma and macrophage interaction in different cancer, which is in the site of the brain. They also use a system partial differential equation but differ in the approach of the modelling situation. These two major works provide the initiative for this study to attempt modifications of their models. Since our focuses on breast cancer, the main concern of this study is referring to Knutsdottir et al. In their model, the production terms for both signals are linear. However, according to Hillen et al. [31] the production rate supposed to be saturates at a certain level. A modification is made to the model and linear stability analysis is carried out to investigate the parameters that contribute to the aggregation.

\section{REVIEW ON EXPERIMENTAL STUDIES}

Experimental studies had been conducted by several researchers regarding on interaction between macrophages and tumor cells. It involved two types of experiments, which are in vitro and in vivo. In vitro studies were carried out in outside living organism such as in petri dish while in vivo studies were carried out inside living organism, usually mice.

The first group that verified macrophages needed for tumor cells motility is van Netten et al. [25]. Through in vitro studies, they found that tumor cells and macrophages aggregate within one day. The details of the interaction were investigated by Lin et al. [26] Using mammary mouse cancer cell lines, they found that CSF-1 is crucial to enhance tumor progression.

Wyckoff et al. [27] then investigate the interaction by inserted mammary tumor, PyMT in mice for two months. At first, $25 \mathrm{nM}$ EGF is placed inside the tumor. After 4 hours, a ratio of three tumor cells to one macrophages are collected. In addition, they found that both tumor cells and macrophages have EGF receptor and CSF-1 receptor respectively. This is the crucial factor for both cells interact continuously.

Motivated by Wyckoff, Goswami et al. [28] further carried out in vitro experiments to show the existence of loop between macrophages and tumor cells. Their findings indicate that the paracrine signalling loop will occur in a short ranged. Patsialou et al. [32] then add a new feature of interaction between both cells which is autocrine signalling. In this interaction, tumor cells secrete and receive its own signal, CSF1.

\section{THE MODEL}

The movement of both tumor cells and macrophages to their chemical signals is known as chemotaxis. Chemotaxis have been studied due to its critical role in biological phenomena [31]. For instance, sperm cells are attracted to chemical substance released by egg cell during fertilization, cell positioning during development of embryo, and migration of immune cell to the site of inflammation. In unicellular organisms, bacteria are the most familiar organism being investigated. Particularly in the movement of E. coli, the life cycle of Dictyostelium discoideum, response to variety of signals for worms, $C$. elegans and others.

A well-developed model was first constructed by Keller and Segel (KS) in 1970 [33]. It has been widely used in models for chemotaxis due to its ability to relate with the real biological situation. They investigate the interactions of chemotactic cells, slime molds and a secreted attractant, cAMP. Results showed that the aggregation formed by cells was regarded as instability. The original model is consisted of four coupled reaction-advection diffusion equations. However, the classical model of $\mathrm{KS}$ is considered complicated to analyze. A minimal version of $\mathrm{KS}$ model has been developed as presented below after several assumptions have been made.

$$
\begin{aligned}
& \frac{\partial u}{\partial t}=\frac{\partial}{\partial x}\left(\mu \frac{\partial u}{\partial x}-\chi u \frac{\partial v}{\partial x}\right)+f(u, v) \\
& \frac{\partial v}{\partial t}=D \frac{\partial^{2} v}{\partial x^{2}}+g(u, v)
\end{aligned}
$$

where $u$ is the cell density, and $c$ is the chemoattractant concentration. $\mu>0$ and $\chi$ is the cell motility and chemotactic sensitivity, respectively. The function $f$ regulates the cell proliferation and death while $g$ represents the production and/or degradation of chemicals. Boundary conditions for this model is often used Neumann boundary condition (no flux). The derivation of this model is based on the Fick's Law and reaction-diffusion equation that can be found in Keshet [34].

This model had been chosen by several researchers for studies on diseases. Luca et al. [35] investigated conditions that lead to aggregation of microglia and formation of local accumulation of chemicals observed in alzheimer's diseases senile plaques. They develop a model for chemotaxis in response to a combination chemoattractant and chemorepellent signalling chemicals. The model consisted of two signalling molecules, $v_{i}, i=1$ and 2 . In their linear stability analysis, the parameter that represents the ratio of effective strengths of attraction and repulsion produce three different regions of pattern formation. Each of the regions is numerically solved to agree with the predictions of linear stability analysis.

Another model by Russell [36] used the KS model to analyze the treatment of a chronic wound with oxygen therapy techniques. Unlike normal wounds, some wounds often linked with diseases such as diabetes which originally occurred at the inflammatory sites for a long period. This model consisted oxygen levels, neutrophil and bacteria concentration. The model was raised to investigate whether it can treated with different time scale and boundary layer problem with two boundaries. A similar work was conducted by Guffey [37] two years later, which included optimal control theory for the problem. With the addition of new chemoattractant concentration released by bacteria, the system consisted of four variable.

The paradox of tumor immunology was initiated by Knutsdottir et al. [29] involving two cells, macrophage and tumor cell together with their own chemical signals. They made use the KS model to describe in vitro experiments about the interaction of both cells. The model involve two types of interaction which are paracrine signalling loop and autocrine signalling loop. The instability of the system is indicated by the aggregation of the cells since this form will make macrophages and tumor cells migrate from their primary sites of infection. Linear stability was conducted to investigate the parameter of the equation that crucial for the aggregation to be formed. Their model consists of four variables presented in Eqns. (3)-(6).

$$
\begin{aligned}
& \frac{\partial M}{\partial t}=\mu \frac{\partial^{2} M}{\partial x^{2}}-\frac{\partial}{\partial x}\left(\chi_{1} M \frac{\partial C}{\partial x}\right) \\
& \frac{\partial T}{\partial t}=\mu \frac{\partial^{2} T}{\partial x^{2}}-\frac{\partial}{\partial x}\left(\chi_{2} T \frac{\partial E}{\partial x}\right)-\frac{\partial}{\partial x}\left(\chi_{3} T \frac{\partial C}{\partial x}\right) \\
& \frac{\partial C}{\partial t}=D \frac{\partial^{2} C}{\partial x^{2}}+b_{1} T-\gamma_{1} C \\
& \frac{\partial E}{\partial t}=D \frac{\partial^{2} E}{\partial x^{2}}+b_{2} M-\gamma_{2} E
\end{aligned}
$$

where $M$ denoted the density of macrophage, $T$ is density of tumor cell, $C$ is concentration of CSF-1 and $E$ is concentration of EGF. Compared to KS model, this equation assumes there is neither birth nor death of the cells $(f=0)$. The chemical signals are assume to be linear in 
production and degradation $(g \neq 0)$. Secretion of CSF-1 and EGF is denoted by $b_{1}$ and $b_{2}$ respectively while $\gamma_{1}$ and $\gamma_{2}$ are its degradation. Parameter $\mu, D$ and $\chi_{i}$ where $i=1,2,3$ are same as in the KS model.

This paper modified the model developed by Knutsdottir et al. [29] In Eqn. (2), the form of $g$ is linear with the density of cells. This means that the production of CSF-1 and EGF increase when both the density of tumor cells and macrophages increase. According to Hillen et al. [31] the production of chemicals does not increase continuously. It supposed to saturate with increasing cell density, which in the form of saturating functions as in Eqn. (7) and Eqn. (8).

$$
\begin{aligned}
& \frac{\partial C}{\partial t}=b_{1}\left(\frac{T}{h_{1}+T}\right) \\
& \frac{\partial E}{\partial t}=b_{2}\left(\frac{M}{h_{2}+M}\right)
\end{aligned}
$$

where $b_{i}$ governs the secretion rate of signals and $h_{i}$ indicates the density of cells for $i=1,2$. Several number of chemotactic models also used this terms which can be found in Maini et al. [38] and Myerscough et al. [39]. A new modified model is presented in Eqn. (9)-(12).

$$
\begin{aligned}
& \frac{\partial M}{\partial t}=\mu \frac{\partial^{2} M}{\partial x^{2}}-\frac{\partial}{\partial x}\left(\chi_{1} M \frac{\partial C}{\partial x}\right) \\
& \frac{\partial T}{\partial t}=\mu \frac{\partial^{2} T}{\partial x^{2}}-\frac{\partial}{\partial x}\left(\chi_{2} T \frac{\partial E}{\partial x}\right)-\frac{\partial}{\partial x}\left(\chi_{3} T \frac{\partial C}{\partial x}\right) \\
& \frac{\partial C}{\partial t}=D \frac{\partial^{2} C}{\partial x^{2}}+b_{1}\left(\frac{T}{h_{1}+T}\right)-\gamma_{1} C \\
& \frac{\partial E}{\partial t}=D \frac{\partial^{2} E}{\partial x^{2}}+b_{2}\left(\frac{M}{h_{2}+M}\right)-\gamma_{2} E
\end{aligned}
$$

with initial and boundary condition

$$
\begin{array}{cc}
M(x, 0)=M_{0}, \quad T(x, 0)=T_{0}, & C(x, 0)=C_{0}, \quad E(x, 0)=E_{0} \\
\left.\left(\mu \frac{\partial M}{\partial x}-\chi_{1} M \frac{\partial C}{\partial x}\right)\right|_{0, L}=0, & \left.\frac{\partial C}{\partial x}\right|_{0, L}=0, \\
\left.\left(\mu \frac{\partial T}{\partial x}-\chi_{2} T \frac{\partial E}{\partial x}-\chi_{3} T \frac{\partial C}{\partial x}\right)\right|_{0, L}=0, & \left.\frac{\partial E}{\partial x}\right|_{0, L}=0 .
\end{array}
$$

Table 1 Parameter values used are obtained in $[29,30,40]$

\begin{tabular}{ccc}
\hline Parameter & Units & Value \\
\hline$\mu$ & $\mu \mathrm{m}^{2} / \mathrm{min}$ & 6 \\
$\chi_{1}, \chi_{2}, \chi_{3}$ & $\mu \mathrm{m}^{2} / \mathrm{min} \mathrm{n}$ & 1000 \\
$b_{1}$ & $\mathrm{nM} / \mathrm{min}$ & 6.9 \\
$b_{2}$ & $\mathrm{nM} / \mathrm{min}$ & 9.6 \\
$\gamma_{1}$ & $1 / \mathrm{min}$ & $3 \times 10^{-2}$ \\
$\gamma_{2}$ & $1 / \mathrm{min}$ & $1.5 \times 10^{-2}$ \\
\hline
\end{tabular}

\section{RESULTS AND DISCUSSION}

In this section, the analysis is presented for two separate cases which are stability analysis for paracrine signalling loop $\left(\chi_{3}=0\right)$ and stability analysis for autocrine signalling loop $\left(\chi_{3} \neq 0\right)$. First, the model in Eqn. (9)-(12) are simplified to yield a smaller number of parameters by transforming the system into dimensionless form. Second, the model is reduced from four equations into two equations by using quasi-steady state assumptions. Lastly, the linear stability analysis is done by using perturbation method.

Stability analysis for paracrine signalling loop $\left(\chi_{3}=0\right)$

Consider the first case, in which the tumor cell does not receive its chemical signal, CSF-1. To nondimensionalize the model in Eqn. (9)(12), let

$$
\begin{array}{lll}
x^{*}=\frac{x}{\widehat{x}}, & t^{*}=\frac{t}{\bar{t}^{\prime}} & M^{*}=\frac{M}{\widehat{M}^{\prime}} \\
T^{*}=\frac{T}{\widehat{T}^{\prime}} & C^{*}=\frac{C}{\hat{C}^{\prime}} & E^{*}=\frac{E}{\hat{E}}
\end{array}
$$

where

$$
\begin{array}{lll}
\hat{x}=\sqrt{\frac{D}{\gamma_{2}}}, & \hat{t}=\frac{D}{\gamma_{2} \mu} & \widehat{M}=h_{2} \\
\hat{T}=h_{1}, & \hat{C}=\frac{b_{1}}{\gamma_{1}}, & \hat{E}=\frac{b_{2}}{\gamma_{2}}
\end{array}
$$

The asterisks $(*)$ denote dimensionless variables and parameters. Note that the choice of scaling parameters and variables in (15) are not unique. Removing $(*)$ from the nondimensionalized variables and parameters for notational simplicity, Eqn. (9)-(12) become

$$
\begin{gathered}
\frac{\partial M}{\partial t}=\frac{\partial^{2} M}{\partial x^{2}}-A_{1} \frac{\partial}{\partial x}\left(M \frac{\partial C}{\partial x}\right) \\
\frac{\partial T}{\partial t}=\frac{\partial^{2} T}{\partial x^{2}}-A_{2} \frac{\partial}{\partial x}\left(T \frac{\partial E}{\partial x}\right) \\
\varepsilon \frac{\partial C}{\partial t}=\frac{\partial^{2} C}{\partial x^{2}}+B^{2}\left(\frac{T}{1+T}-C\right) \\
\varepsilon \frac{\partial E}{\partial t}=\frac{\partial^{2} E}{\partial x^{2}}+\frac{M}{1+M}-E
\end{gathered}
$$

where the new parameter are represent as

$$
A_{1}=\frac{\chi_{1} b_{1}}{\gamma_{1} \mu}, \quad A_{2}=\frac{\chi_{2} b_{2}}{\gamma_{2} \mu}, \quad \varepsilon=\frac{\mu}{D^{\prime}}, \quad B^{2}=\frac{\gamma_{1}}{\gamma_{2}}
$$

and the steady state is non-trivial as in (21).

$$
(M, T, C, E)=\left(\widetilde{M}, \widetilde{T}, \frac{\widetilde{T}}{1+\widetilde{T}}, \frac{\widetilde{M}}{1+\widetilde{M}}\right)
$$

$\widetilde{M}$ and $\tilde{T}$ are the average density of macrophages and tumor cells. Notice that the value of $\varepsilon$ is very small as in Eqn. (18) and (19). This is due to the signalling molecule diffuse faster than the random motion of the cells which can be known as quasi-steady state approximations. By making use of this approximations, Eqn. (18) and Eqn. (19) becomes

$$
\begin{aligned}
& 0 \approx \frac{\partial^{2} C}{\partial x^{2}}+B^{2}\left(\frac{T}{1+T}-C\right) \\
& 0 \approx \frac{\partial^{2} E}{\partial x^{2}}+\frac{M}{1+M}-E
\end{aligned}
$$

Following from Knutsdottir [29], Luca [35] and Maini [38], Green function is used to obtain the solution for Eqn. (22) and Eqn. (23).

$$
C=\frac{B}{2} \int_{-\infty}^{\infty} e^{-B\left|x-x^{\prime}\right|} \frac{T}{1+T} d x^{\prime} \equiv K_{1} * \frac{T}{1+T}
$$




$$
E=\frac{1}{2} \int_{-\infty}^{\infty} e^{-\left|x-x^{\prime}\right|} \frac{M}{1+M} d x^{\prime} \equiv K_{2} * \frac{M}{1+M}
$$

where $K_{1}$ and $K_{2}$ is the kernel that represent by $e^{-B|x|}$ and $e^{-|x|}$ respectively. Eqn. (24) and (25) contain convolution integral terms which now can reduced from 4 PDE's to 2 PDE's system of equations. Substituting these equation into Eqn. (16) and (17) yields

$$
\begin{gathered}
\frac{\partial M}{\partial t}=\frac{\partial^{2} M}{\partial x^{2}}-A_{1} \frac{\partial}{\partial x}\left[M \frac{\partial}{\partial x}\left(K_{1} *\left(\frac{T}{1+T}\right)\right)\right] \\
\frac{\partial T}{\partial t}=\frac{\partial^{2} T}{\partial x^{2}}-A_{2} \frac{\partial}{\partial x}\left[T \frac{\partial}{\partial x}\left(K_{2} *\left(\frac{M}{1+M}\right)\right)\right]
\end{gathered}
$$

Stability analysis can be performed on this reduced model to see if a small perturbation from the steady state can cause spontaneous aggregation of the cells. The following perturbation is introduced into the system:

$$
M=\widetilde{M}+M_{0} e^{i q x+\sigma t} \quad T=\tilde{T}+T_{0} e^{i q x+\sigma t}
$$

where $q$ is the wavenumber of the perturbation and $\sigma$ is the growth rate of the perturbation. Introducing this perturbation into the system and it will lead to the system of equation $\mathbf{A x}=\mathbf{b}$, where $\mathbf{A}$ :

$$
\mathbf{A}=\left(\begin{array}{cc}
\sigma+q^{2} & -\frac{q^{2} A_{1} B^{2} \widetilde{M}}{(1+\widetilde{T})^{2}\left(B^{2}+q^{2}\right)} \\
-\frac{q^{2} A_{2} \tilde{T}}{(1+\widetilde{M})^{2}\left(1+q^{2}\right)} & \sigma+q^{2}
\end{array}\right)
$$

For non-trivial solutions $\left(M_{0}, T_{0} \neq 0\right)$, the determinant of $\mathbf{A}$ needs to be zero, $\operatorname{det}(\mathbf{A})=0$. Then, it yields equation in the form of $\sigma^{2}+p \sigma+$ $r=0$, where

$$
\begin{gathered}
p=2 q^{2} \\
r=q^{4}\left(1-\frac{A_{1} A_{2} B^{2} \widetilde{M} \tilde{T}}{(\widetilde{T}+1)^{2}(\widetilde{M}+1)^{2}\left(1+q^{2}\right)\left(B^{2}+q^{2}\right)}\right)
\end{gathered}
$$

For spontaneous aggregation to occur, the small perturbations have to cause instability in the system. This is true when the growth rate, $\sigma>0$ and $r<0$.

$$
f\left(q^{2}\right) \equiv\left(1+q^{2}\right)\left(B^{2}+q^{2}\right)<\frac{A_{1} A_{2} B^{2} \widetilde{M} \tilde{T}}{(\widetilde{M}+1)(\tilde{T}+1)} \equiv Z
$$

The inequality (32) provides the range of parameter that can cause aggregation in response to small perturbations from the steady state. It shows large value of wavenumbers, $q$ will not lead to instability. Besides, the aggregation for both macrophages and tumor cells are depend on $A_{1}$ and $A_{2}$, provided both are sufficiently large. Recall that the related parameter contain in $A_{i}$ are $\chi_{i}, b_{i}$, and $\gamma_{i}$. Hence, the chemotaxis parameter together with secretion rate should be inhibited while increasing the degradation rate can prevent the aggregation from being occur

\section{Stability analysis for paracrine and autocrine signalling loop $\left(\chi_{3} \neq 0\right)$}

In this case, tumor cell will release CSF-1 and receive this signal. The new matrix $\mathbf{A}$ for this system is

$$
\left(\begin{array}{cc}
\sigma+q^{2} & -\frac{q^{2} A_{1} B^{2} \widetilde{M}}{(1+\tilde{T})^{2}\left(B^{2}+q^{2}\right)} \\
-\frac{q^{2} A_{2}}{(1+\widetilde{M})^{2}\left(1+q^{2}\right)} & \sigma+q^{2}-\frac{A_{3} \tilde{T} B^{2} q^{2}}{(\tilde{T}+1)^{2}\left(B^{2}+q^{2}\right)}
\end{array}\right)
$$

Finding the $\operatorname{det}(\mathbf{A})=0$, it will leads to a new inequality in Eqn. (34)

$$
\begin{aligned}
& f\left(q^{2}\right)=\left(1+q^{2}\right)\left(B^{2}+q^{2}\right)-\frac{q^{2} A_{3} B^{2} \tilde{T}}{(\widetilde{T}+1)^{2}} \\
&<\frac{A_{1} A_{2} B^{2} \tilde{T} \widetilde{M}}{(\widetilde{T}+1)^{2}(\widetilde{M}+1)^{2}}+\frac{A_{3} B^{2} \tilde{T}}{(\widetilde{T}+1)^{2}} \equiv Z_{2}
\end{aligned}
$$

Comparing inequality in Eqn. (34) and (32), the addition of autocrine signalling will increase the possibility of aggregation to be occur. This due to the high concentration of CSF-1 will attract macrophages to interact with tumor cells.

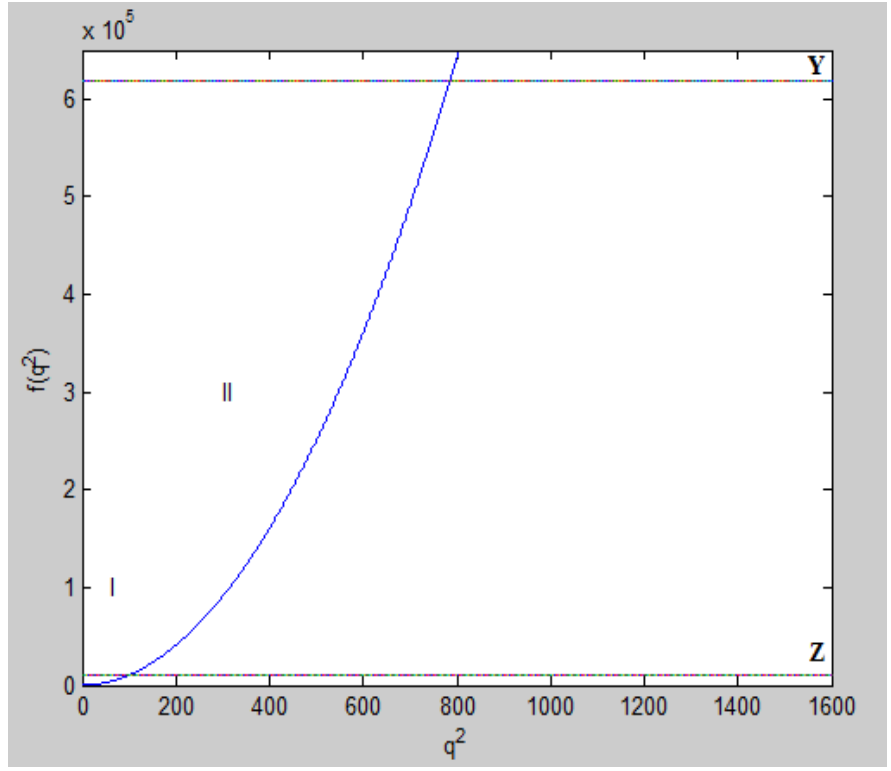

Fig. 2 The solid blue line shows the functions $f\left(q^{2}\right)$ from Eqn. (32). Typical value of $Z$ is represent by lower dotted line while typical value of $Y$ which obtained from Knutsdottir model is represent by upper dotted line. The figure implies that any value of $Z$ always produce smaller region of instability $\mathrm{I}$, compared to the value of $Y$ that produce larger region of instability II.

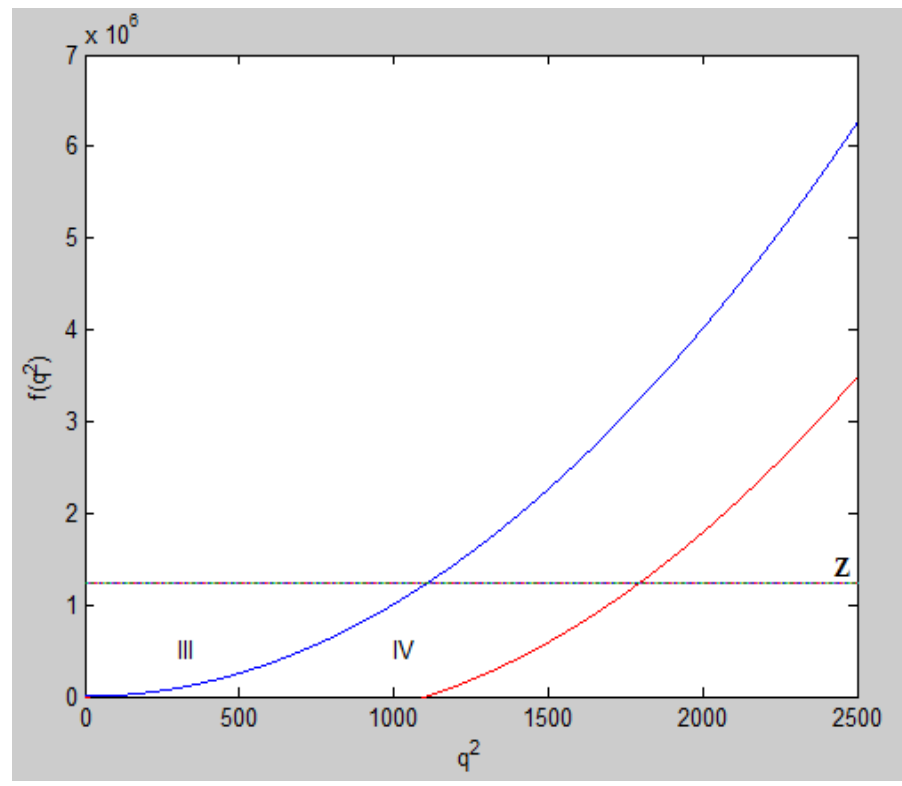

Fig. 3 The solid blue line shows the functions $f\left(q^{2}\right)$ from Eqn. (32) while the solid red line shows the functions $f\left(q^{2}\right)$ from Eqn. (34). The figure illustrates the inclusion of autocrine signalling loop produces a large instability region (III and IV) compared with the paracrine signalling loop alone. 


\section{CONCLUSION}

The interaction between macrophages and tumor cells in breast cancer can be modelled by system of 4 PDE's that incorporate chemotaxis and reaction-diffusion equations. This work proposed different secretion terms that in the form of saturating functions. From the stability analysis, the secretion rate, chemotaxis sensitivity and degradation rate are crucial in determining the occurrence of aggregations for both cases which are paracrine signalling loop and the inclusion of autocrine signalling loop. Besides, stability region in Fig. 2 illustrates the region of instability is reduced compared with the previous model proposed by Knutsdottir et al. [29]. In addition, the inclusion of autocrine signalling loop increase the possibility of the aggregation to be occurred.

\section{ACKNOWLEDGEMENT}

The authors are thankful to Research Management Centre (UTM) and Ministry of Higher Education (MOHE), Malaysia for financial support through research grants of vote 4F797 and vote 4L854.

\section{REFERENCES}

[1] Wilking, N., Kasteng, F., Bergh, J., Jonsson, B., Kossler, I., Martin, M., Maranon, G., Normand, C., Reed, L., and Widdershoven, G. (2009). A Review of Breast Cancer Care and Outcomes in 18 Countries in Europe, Asia, and Latin America. Retrieved from Comparator Report website: http://www.comparatorreports.se/A_review_of_breast_cancer_care_and outcomes_260ct2009.pdf

[2] Mayer, M., Hunis, A., Oratz, R., Glennon, C., Spicer, P., Caplan E., and Fallowfield, L. (2010). Living with metastatic breast cancer: A global patient survey, Community Oncology, 7, 406-412.

[3] Althuis, M. D., Dozier, J. M., Anderson, W. F., Devesa, S. S., and Brinton, L. A. (2005). Global trends in breast cancer incidence and mortality 1973-1997, International Jounal of Epidemiology, 34, 405-412.

[4] Polyak, K., (2007). Breast cancer: origins and evolution, The Journal of Clinical Investigation, 117, 3155-3163.

[5] Koren, S. and Alj, M. B. (2015). Breast tumor heterogeneity: Source of fitness, hurdle for therapy, Molecular Cell, 60, 537-546.

[6] Gunduz, M. and Gunduz, E. (2011). Breast cancer - Focusing tumor microenvironment, stem cell and metastasis. Croatia: InTech, 69-84.

[7] Enderling, H., Alexander, R. A., Anderson, M. A., Chaplain, J., Munro, A. J., and Vaidya. (2006). Mathematical modelling of radiotherapy strategies for early breast cancer, Journal of Theoretical Biology, 241, 158-171.

[8] Cameron, D. A. (1997). Mathematical modelling of the Response of Breast Cancer to Drug Therapy, Journal of Theoretical Medicine, 2, 137 151 .

[9] Zhang, X., Fang, Y., Zhao, Y., and Zheng, W. (2014). Mathematical modelling the pathway of human breast cancer, Mathematical Biosciences, 253, 25-29.

[10] Kim, Y., and Othmer. H. G. (2013). A hybrid model of tumor-stromal interactions in a breast cancer, Bulletin of Mathematical Biology, 75, 1304-1350.

[11] McDuffie, M. (2014). A hormone therapy model for breast cancer using linear cancer networks. Rose-Hulman Undergraduate Mathematics Journal, 15, 144-156.

[12] Elgert, K. D., Alleva, D. G., and Mullins, D. W. (1998). Tumor-induce immune dysfunction: the macrophage connection. Journal Leukocyte Biology, 64, 275-290.

[13] Lewis, C. E., and Pollard, J. W. (2006). Distinct role of macrophages in different tumor microenvironments, Cancer Research, 66, 605-612.

[14] Condeelis, J. and Pollard, J. W. (2006). Macrophages: Obligate partners for tumor cell migration, invasion and metastasis, Cell, 124, 263-266.

[15] Wynn, T. A., Chawla, A., and Pollard, J. W. (2013). Macrophage biology in development, homeostasis and disease, Nature, 496, 445-455.

[16] Qian, B. Z. and Pollard, J. W. (2010). Macrophage diversity enhances tumor progression and metastasis, Cell, 141, 39-51.

[17] Leek, R. D. and Harris, A. L. (2002). Tumor-associated macrophages in breast cancer, Journal of Mammary Gland Biology and Neoplasia, 7, 177 189.

[18] van der Bij, G. J., Oosterling, S. J., Meijer, S., Beelen, R. H. J., and van Egmond, M. (2005). The role of macrophages in tumor development. Cellular Oncology, 27, 203-213.
[19] Hao, N. B., Lu, M. H., Fan, Y. H., Cao, L. H., Zhang, Z. R., and Yang, S. M. (2012). Macrophages in tumor microenvironments and the progression of tumors, Clinical and Developmental Immunology, 2012, $1-11$.

[20] Sousa, S., Brion, R., Lintunen, M., Kronqvist, P., Sandholm, P., Monkkonen, J., Lehtinen, P. L., Lauttia, S., Tynninen, O., Joensuu, H., Heymann, D., and Maatta, J. A. (2015). Human breast cancer cells educate macrophages toward the M2 activation status, Breast Cancer Research, 17, 101-124.

[21] Italiani, P. and Boraschi, D. (2015). New insights into tissue macrophages: from their origin to the development of memory, Immune Network, 15, 167-176.

[22] Sica, A. and Mantovani, A. (2012). Macrophage plasticity and polarization: in vivo veritas, Journal of Clinical Investigation, 122, 787795.

[23] Bingle, L., Brown, N. J. and Lewis, C. E. (2002). The role of tumor associated macrophages in tumor progression: Implications for new anticancer therapies, Journal of Pathology, 196, 254-265.

[24] Mills, C. D., Kincaid, K., Alt, J. M., Heilma, M. J., and Hill, A. M. (2000). M1/M2 Macrophages and the Th1/Th2 paradigm, The Journal of Immunology, 164, 6166-6173.

[25] van Netten, J. P., Ashmead, B. J., Parker, R. I., Thornton, I. G., Fletcher, C., Cavers, D., Coy, P., and Brigden, M. L. (1993). Macrophage-tumor cell associations: A factor in metastasis of breast cancer?, Journal of Leukocyte Biology. 54, 360-362.

[26] Lin, E. Y., Nguyen, A. V., Russell, R. G., and Pollard, J. W. (2001). Colony-stimulating factor 1 promotes progression of mammary tumors to malignancy, Journal Experimental Medicine, 193, 727-739.

[27] Wyckoff, J., Wang, W., Lin, E. Y., Wang, Y., Pixley, F., Stanley, E. R., Graf, T., Pollard, J. W., Segall J., and Condeelis, J. (2004). A paracrine loop between tumor cells and macrophages is required for cell migration in mammary tumors, Cancer Research, 64, 7022-7029.

[28] Goswami, S., Sahai, E., Wyckoff, J. B., Cammer, M., Cox,D., Pixley, F. J., Stanley, E. R., Segall, J. E., and Condeelis. J. S. (2005). Macrophages promote the invasion of breast carcinoma cells via colony stimulating factor-1/epidermal growth factor paracrine loop, Cancer Research, 65, 5278-5283.

[29] Knutsdottir, H., Palsson, E., and Edelstein-Keshet, L. (2014). Mathematical model of macrophage-facilitated breast cancer cells invasion, Journal of Theoretical Biology, 357, 184-199.

[30] Elitas, M. and Zeinali, S. (2016). Modeling and simulation of EGF-CSF1 pathway to investigate glioma-macrophage interaction in brain tumors, International Journal of Cancer Study and Research, S5, 1-8.

[31] Hillen, T. and Painter. K. J. (2009). A user's guide to PDE models for chemotaxis, Journal of Mathematical Biology, 58, 183-217.

[32] Patsialou, A., Wyckoff, J., Wang, Y., Goswami, S., Stanley, E. R., and Condeelis, J. S., (2009). Invasion of human breast cancer cells in vivo requires both paracrine and autocrine loops involving the colonystimulating factor-1 receptor, Cancer Research, 69, 9498-9506.

[33] Keller, E. F. and Segel, L. E. (1971). Model for Chemotaxis, Journal of Theoretical Biology, 30, 225-234.

[34] Keshet, L. E. (1976). Mathematical Models in Biology, McGraw-Hill, Inc., 393-395.

[35] Luca, M., Ross, A. C., Keshet, L. E., and Mogilner, A. (2003). Chemotactic Signaling, Microglia, and Alzheimer's Disease Senile Plaques: Is there a Connection? Bulletin of Mathematical Biology, 65, 693-730.

[36] Russell., B. C. (2013). Using partial differential equations to model and analyze the treatment of a chronic wound with oxygen therapy techniques. Honors College Capstone Experience/Thesis Projects. Western Kentucky University.

[37] Guffey, S. (2015). Application of a numerical method and optimal control theory to a partial differential equation model for a bacterial infection in a chronic wound. Masters Theses \& Specialist Projects. Western Kentucky University.

[38] Maini, P. K., Myerscough, M. R., Winters, K. H and Murray, J. D. (1991). Bifurcating spatially heterogeneous solutions in a chemotaxis model for biological pattern generation. Bulletin of Mathematical Biology, 53, 701719.

[39] Myerscough, M. R., Maini, P. K., and Painter, K. J. (1998). Pattern Formation in a Generalized Chemotactic Model, Bulletin of Mathematical Biology, 60, 1-26.

[40] Knutsdottir, H., Condeelis, J. S., and Palsson. E. (2016). 3-D individual cell based computational modelling of tumor cell-macrophage paracrine signalling mediated by EGF and CSF-1 gradients, Integrative Biology, 8, 104-119. 